\section{Screening for coronary artery calcium in a high-risk population: the ROBINSCA trial}

\author{
Sabine JAM Denissen', Carlijn M van der Aalst', \\ Marleen Vonder ${ }^{2}$, Jan Willem C Gratama ${ }^{3}$, Henk J Adriaansen ${ }^{4}$, \\ Dirkjan Kuijpers ${ }^{5,6}$, Jeanine E Roeters van Lennep ${ }^{7}$, \\ Rozemarijn Vliegenthart ${ }^{2}$, Pim van der Harst ${ }^{2,8,9}$, \\ Richard L Braam ${ }^{10}$, Paul RM van Dijkman ${ }^{11,12}$, \\ Matthijs Oudkerk ${ }^{13,14}$ and Harry J de Koning '
}

European Journal of Preventive Cardiology $0(0) 1-5$

(C) The Author(s) 2020 (c) (1) (9)

Article reuse guidelines: sagepub.com/journals-permissions DOI: $10.1177 / 2047487320932263$ journals.sagepub.com/home/cpr

(S)AGE
Cardiovascular disease (CVD) remains the main cause of death worldwide, accounting for $44 \%$ of all noncommunicable disease deaths, of which most are attributable to coronary heart disease (CHD). ${ }^{1}$ Coronary artery calcification (CAC) has a strong association with major cardiovascular events and mortality, and has a high risk-predictive value of CHD in asymptomatic individuals. ${ }^{2,3}$ It has been argued that the amount of CAC, expressed in the CAC score, can be used in population-based screening.

The Dutch Risk Or Benefit IN Screening for CArdiovascular disease (ROBINSCA) trial is the first large-scale population-based randomised controlled trial (RCT) to investigate whether CAC screening followed by preventive treatment is effective in reducing CHD-related morbidity and mortality in asymptomatic individuals. ${ }^{4,5}$ The aim of this study was to investigate the CAC prevalence and predictors in the ROBINSCA trial, which included an asymptomatic high-risk potential target population from the general population.

The rationale and design of the ROBINSCA trial have been described before. ${ }^{5}$ Briefly, 43,447 potentially high-risk women (55-74 years) and men (45-74 years) from the national population registry who completed a baseline questionnaire to assess sociodemographic and health characteristics and gave informed consent were randomly allocated (1:1:1) to either the control arm, intervention arm A (screening according to traditional risk factors) or intervention arm B (CAC screening). The current study focuses on the CAC screening arm (Figure 1). The Minister of Health authorised the ROBINSCA trial in 2013.

CAC screening was performed using computed tomography scanning to identify CVD risk according to the CAC score, which represents the total amount of any CAC. ${ }^{6}$ CAC scores were categorised into low (Agatston 0-99), high (Agatston 100-399) and very high (Agatston $\geq 400$ ) risk. ${ }^{2}$

The effects of baseline characteristics on CAC score were analysed using a two-step approach regression analyses for modelling presence, both any CAC

\footnotetext{
'Department of Public Health, Erasmus Medical Centre, The Netherlands ${ }^{2}$ Centre for Medical Imaging North-East Netherlands (CMI-NEN), University Medical Centre Groningen, The Netherlands

${ }^{3}$ Department of Radiology and Nuclear Medicine, Gelre Hospitals, The Netherlands

${ }^{4}$ Clinical Chemistry and Hematology Laboratory, Gelre Hospitals, The Netherlands

${ }^{5}$ Department of Radiology, University Medical Center Groningen, The Netherlands

${ }^{6}$ Department of Radiology, Haaglanden Medical Centre Bronovo,

The Netherlands

${ }^{7}$ Department of Internal Medicine, Erasmus Medical Centre,

The Netherlands

${ }^{8}$ Department of Cardiology, University Medical Centre Groningen, The Netherlands

${ }^{9}$ Department of Cardiology, University Medical Centre Utrecht, The Netherlands

${ }^{10}$ Department of Cardiology, Gelre Hospitals, The Netherlands

"Department of Cardiology, Leids University Medical Centre,

The Netherlands

${ }^{12}$ Department of Cardiology, Haaglanden Medical Centre Bronovo,

The Netherlands

${ }^{13}$ University of Groningen, University Medical Centre Groningen,

The Netherlands

${ }^{14}$ Institute for Diagnostic Accuracy - iDNA, The Netherlands

The first two authors contributed equally.

Trial registration number: NTR647I.
}

\section{Corresponding author:}

Sabine Denissen, Department of Public Health, Erasmus MC, P.O. Box 2040, 3000 CA Rotterdam, The Netherlands.

Email: s.denissen@erasmusmc.nl 


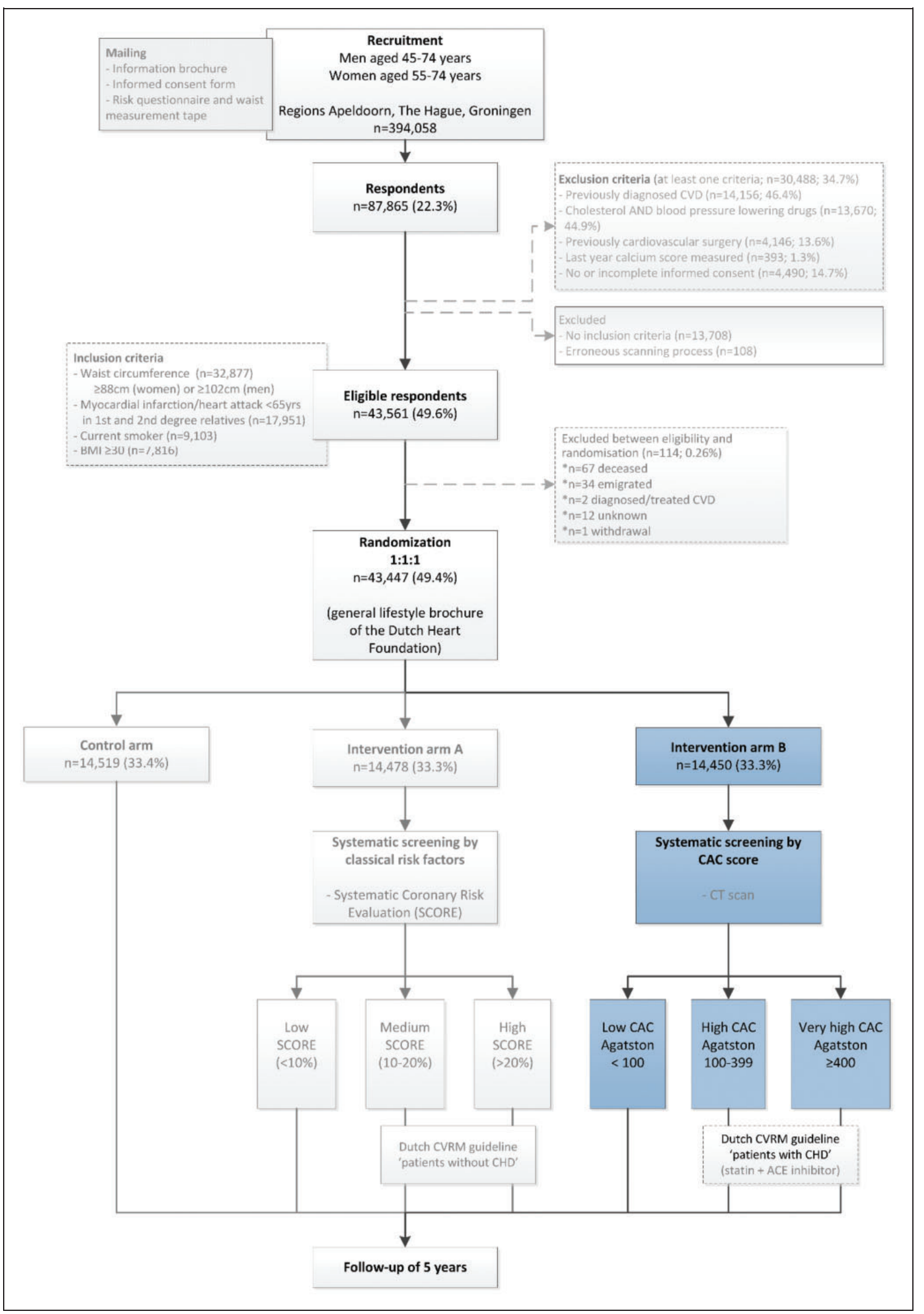

Figure I. Flowchart of the ROBINSCA trial study design in which CAC scoring is performed in intervention arm B. BMI: body mass index; CAC: coronary artery calcium; CHD: coronary heart disease; CT: computed tomography; CVD: cardiovascular disease; CVRM: cardiovascular risk management; SCORE: systematic coronary risk evaluation. 
and CAC score of 400 or higher (multivariable backward logistic regression), and extent (multivariable backward linear regression of the log-transformed CAC score) of CAC in women and men separately. Variables included in the models were age, educational level, waist circumference cut-off ( $88 \mathrm{~cm}$ for women and $102 \mathrm{~cm}$ for men), body mass index (BMI) cut-off $\left(30 \mathrm{~kg} / \mathrm{m}^{2}\right)$, family history of CHD, smoking, diabetes mellitus, hypertension and/or hypercholesterolemia in the past year, and baseline use of either antihypertensive or lipid-lowering medication (according to selfreported data from the baseline questionnaire). A $P$ value of less than 0.05 was considered statistically significant. All analyses were performed using IBM SPSS Statistics version 24.0.

Of the 12,950 screened participants, $48.1 \%$ were women and $94.2 \%$ were born in The Netherlands. The median age was 64 years in women and 62 years in men. Regarding CVD risk factors, $20.0 \%$ were current smokers at baseline, $3.4 \%$ reported diabetes mellitus, $16.4 \%$ and $15.3 \%$ reported being diagnosed with hypertension and hypercholesterolemia, respectively, in the year before baseline, and $44.9 \%$ reported a family history of CHD.

CAC was absent in $39.2 \%$ of the total study population. Overall, $48 \%$ of women had a zero CAC score compared to $20.7 \%$ of men in the same age category and $31.2 \%$ of all men. Furthermore, $16.8 \%$ of women had a CAC score of 100 or higher compared to $40.0 \%$ of men in the same age category and $30.7 \%$ of all men. The CAC distribution in the ROBINSCA trial is compared to the German Heinz Nixdorf Recall Study and the American Multi Ethnic Study of Atherosclerosis in the Supplementary material.

Age, high waist circumference, family history of CHD, smoking at baseline, diabetes mellitus, self-reported hypertension or hypercholesterolemia at baseline and baseline use of either antihypertensive or lipid-lowering medication were all selected as predictors in the backward regression analysis of the presence of CAC and CAC of 400 or greater, and in the linear regression for predicting the log-transformed CAC extent in women (Table 1). Age, educational level, high BMI, family history of CHD, smoking at baseline, diabetes mellitus, self-reported hypercholesterolemia at baseline and baseline use of either antihypertensive or lipid-lowering medication were selected as predictors in the analyses for men (Table 1). A higher educational level predicted a lower CAC score in men. The composition of the predictors differed moderately in the models for women and men.

The associations of age, male sex, diabetes mellitus and smoking with higher CAC scores are well known. ${ }^{7}$ A lower socioeconomic status, indicated by educational level, significantly predicted a higher extent of CAC in men. This association is possibly a result of a less favourable lifestyle in terms of smoking, diet and physical activity. ${ }^{8}$ Diabetes mellitus was one of the strongest predictors of CAC presence in women. This is in line with previous research in which diabetes mellitus was identified to have a greater impact in women compared to men. ${ }^{9}$ Moreover, diabetes mellitus was a strong predictor for CAC extent in both sexes, suggesting that it is the most important risk factor for CAC development after sex and age. Regarding BMI and waist circumference, our results confirm earlier findings that BMI is not a strong predictor for the presence of $\mathrm{CAC}$, while waist circumference is more predictive of CAC presence. ${ }^{10}$ The predictive value of the baseline use of either antihypertensive or lipid-lowering medication in CAC development was also seen in previous research. However, statins have been associated with increased CAC scores, but not with more CVD events. It is suggested that statins induce CAC progression and, at the same time, plaque repair. ${ }^{11}$

This study contributes to evidence on identifying the optimal target population for screening from the general population that will gain most healthy life-years from screening and subsequent treatment. All inclusion criteria for the ROBINSCA trial (smoking, waist circumference, BMI and a family history of CHD) were statistically significant predictors of CAC. Future analyses should provide evidence on whether the study population includes individuals who benefit most.

A main limitation is that the ROBINSCA population is not representative of all ethnic groups as a result of a homogeneous distribution, although ethnicity is known to affect CAC prevalence and severity. Another possible limitation is that study participants tend to be generally healthier than similar individuals not responding to the participation invitation (healthy volunteer effect). However, the inclusion and exclusion criteria should have minimised this effect. Furthermore, participants using both cholesterollowering and antihypertensive medication were excluded from the trial, which might have affected the found associations of CAC with CVD medication. Finally, baseline data were obtained using a selfreported questionnaire, rather than diagnostic test measures, and might entail some inaccuracies.

In conclusion, this currently largest populationbased RCT for CAC screening in asymptomatic middle-aged Caucasian individuals showed that $30.7 \%$ of men and $16.8 \%$ of women with a CAC score of 100 or greater urgently require preventive treatment. To a large extent, male sex and increasing age, followed by diabetes mellitus and smoking, influence CAC distribution. These results can therefore help determine the best risk prediction and prevention 


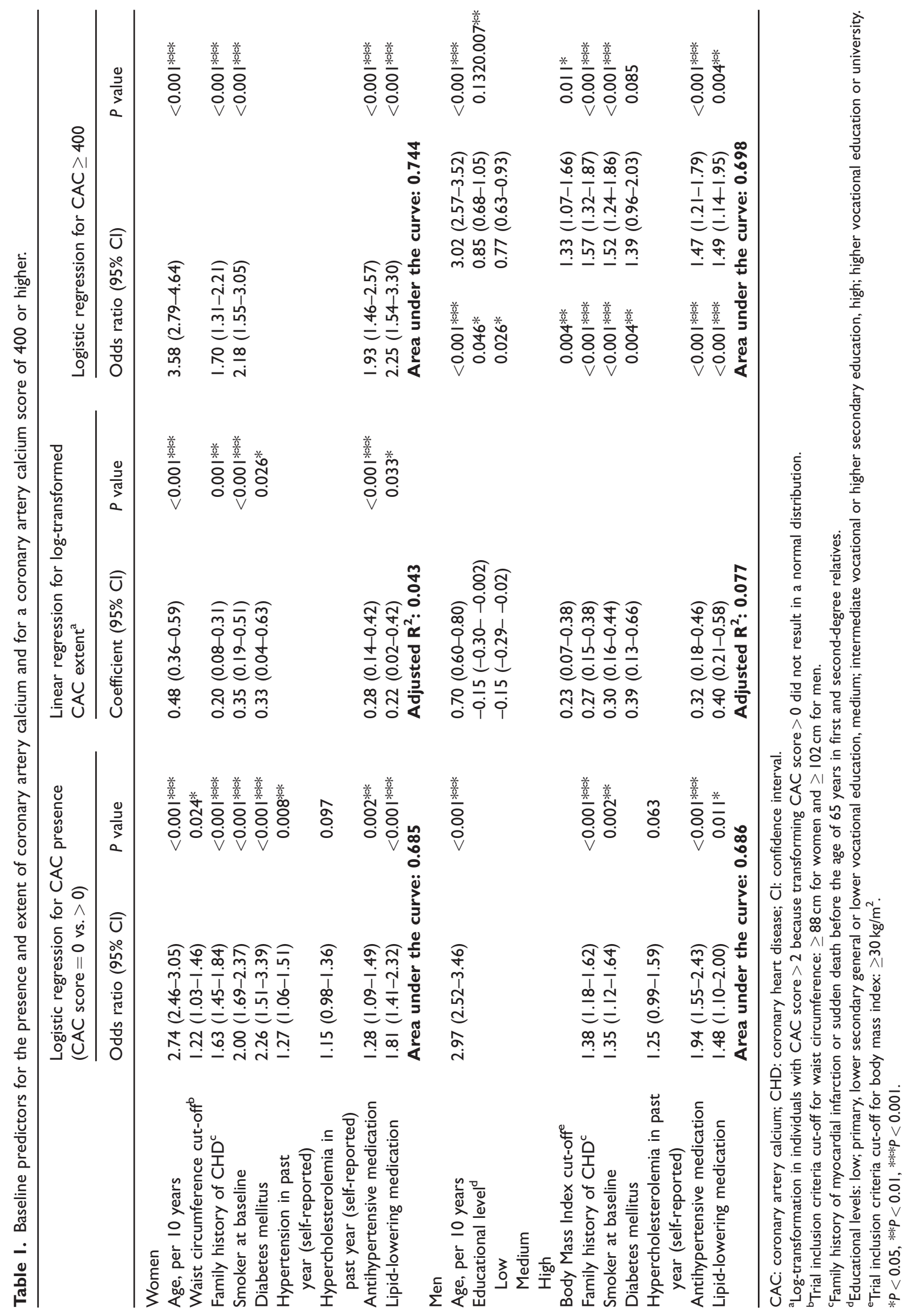


strategy should screening for a high risk of developing CVD be (cost)-effective.

\section{Acknowledgements}

The author(s) would like to thank the European Union for funding the ROBINSCA trial and the Ministry of Health, Welfare and Sports for the ethical approval to perform the trial. Naturally, The author(s) thank all participants for their participation. Furthermore, they would like to thank M Quak for the extensive research assistance, RADventure for developing the data management system and the IVA group for handling all questionnaires and letters. Finally, they thank all employees of the radiology departments of the screening centres (Gelre Hospital Apeldoorn, Bronovo Hospital The Hague and University Medical Centre Groningen) for scanning participants.

\section{Declaration of conflicting interests}

The author(s) declared no potential conflicts of interest with respect to the research, authorship, and/or publication of this article.

\section{Funding}

The author(s) disclosed receipt of the following financial support for the research, authorship, and/or publication of this article: The ROBINSCA trial was funded by an advanced grant (agreement no. 294604) of the European Research Council.

\section{Supplemental material}

Supplemental material for this article is available online.

\section{References}

1. Wilkins E, Wilson L, Wickramasinghe K, et al. European Cardiovascular Disease Statistics 2017. Brussels: European Heart Network, 2017.
2. Greenland P, Blaha MJ, Budoff MJ et al. Coronary calcium score and cardiovascular risk. J Am Coll Cardiol 2018; 72: 434-447.

3. Budoff MJ, Young R, Burke G, et al. Ten-year association of coronary artery calcium with atherosclerotic cardiovascular disease (ASCVD) events: the multi-ethnic study of atherosclerosis (MESA). Eur Heart J 2018; 39: 2401-2408.

4. McEvoy JW, Martin SS, Blaha MJ, et al. The case for and against a coronary artery calcium trial: means, motive, and opportunity. JACC Cardiovasc Imaging 2016; 9: 994-1002.

5. Van der Aalst CM, Vonder M, Gratama JM, et al. Risk or Benefit in Screening for Cardiovascular Disease (ROBINSCA): the rationale and study design of a population-based randomized-controlled screening trial for cardiovascular disease. J Clin Trials 2019; 9: 1000361.

6. Vonder M, van der Aalst CM, Vliegenthart $\mathrm{R}$, et al. Coronary artery calcium imaging in the ROBINSCA Trial: rationale, design, and technical background. Acad Radiol 2018; 25: 118-128.

7. Pletcher MJ, Sibley CT, Pignone M, et al. Interpretation of the coronary artery calcium score in combination with conventional cardiovascular risk factors: the MultiEthnic Study of Atherosclerosis (MESA). Circulation 2013; 128: 1076-1084.

8. Djekic D, Angeras O, Lappas G, et al. Impact of socioeconomic status on coronary artery calcification. Eur $J$ Prev Cardiol 2018; 25: 1756-1764.

9. Nicoll R, Wiklund U, Zhao Y, et al. Gender and age effects on risk factor-based prediction of coronary artery calcium in symptomatic patients: a Euro-CCAD study. Atherosclerosis 2016; 252: 32-39.

10. Roy SK, Zeb I, Kadakia J, et al. Body surface area is a predictor of coronary artery calcium, whereas body mass index is not. Coron Artery Dis 2012; 23: 113-117.

11. Henein M, Granasen G, Wiklund U, et al. High dose and long-term statin therapy accelerate coronary artery calcification. Int J Cardiol 2015; 184: 581-586. 